\title{
Fish fingers on the menu
}

\author{
Zebrafish researchers rejoice! Reverse \\ genetics is now on the menu, thanks \\ to zinc-finger nucleases. \\ "It has been very difficult to do \\ reverse genetics in fish," says University \\ of California, Berkeley-based Sharon \\ Amacher, summing up the frustra- \\ tion of a community of researchers. \\ Indeed, their model's unique advan- \\ tages for imaging and screening have \\ been somewhat offset by this "loss-of- \\ function limitation."
}

Two studies, published in Nature Biotechnology, have now demonstrated that a zebrafish allele of interest can be efficiently disrupted by injecting RNA encoding specific zinc-finger nucleases (ZFNs) into the onecell-stage embryo (Meng et al., 2008; Doyon et al., 2008). Some fish then transmit the mutation through the germline (Fig. 1).

Both studies are the fruits of collaborations between a lab with zebrafish expertise and one skilled at making ZFNs. At the University of Massachusetts Medical School, zinc finger aficionado Scot Wolfe joined forces with his neighbor Nathan Lawson. "It seemed a natural way to bring together the strengths of our two labs to try to do something a bit different," says Wolfe. In Berkeley, Amacher teamed up with her teaching colleague Fyodor Urnov and his group at Sangamo Biosciences to find the right nucleases for her fish studies. "This is a terrific example of collaboration between academia and industry," she says; "it has been incredibly productive."

The fact that both breakthroughs come from such team efforts is no coincidence. Rather, it reflects the complexity of making ZFNs. The nuclease specificity for a DNA sequence is conferred by several zinc-finger peptides, each of which recognizes a triplet of nucleic acids. But making a ZFN by modular assembly of peptides that recognize consecutive triplets of a target sequence does not always work, as highlighted recently by Keith Joung (Ramirez et al., 2008). "A small subset of zinc fingers are 'mix-and-matchable,"

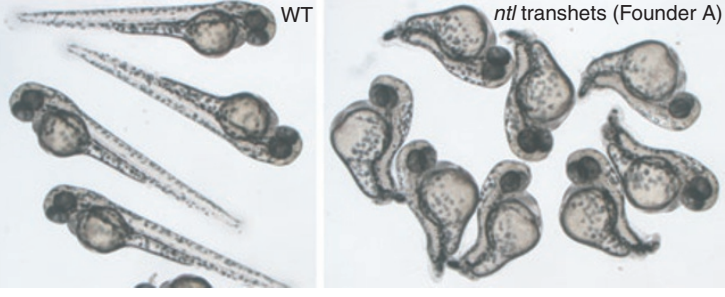

They selected zinc fingers with the desired specificity using a robust one-hybrid bacterial assay to screen libraries of motifs in which some elements of design — amino acids known to be important for specific sequence recognition-had been integrated. Although at the moment the Wolfe and Lawson team recommends this combination of design and selection, they want to use their experimental system to learn more about which zinc fingers work together, hoping to improve the explains Urnov, referring to Joung's report. "However, as soon as you stop trying to recognize things that have the GNN motif, then the 'mix-and-matchability' of individual zinc fingers really plummets."

To address this difficulty, the two teams took fundamentally different approaches. Amacher's study has used the archive of prevalidated 2-zinc-finger modules built by Sangamo. According to Urnov, using such tandems instead of individual fingers addresses the modular assembly problem by taking into account the structural connectivity between adjacent zinc-finger peptides. Based on the modules with characterized specificity in the Sangamo archive, Amacher and Urnov designed several pairs of 4-zincfinger nucleases per target gene. They then used an elegant yeast-based assay to identify the subset of nucleases that work in fish. For anyone interested in taking this approach, ZFNs built based on the Sangamo archive can be obtained via Sigma-Aldrich, and the yeast validation assay is freely available.

Wolfe and Lawson adopted a different strategy - a combination of design and selectionto produce zinc fingers with new specificity and assemble them into 3-zinc-finger nucleases that recognize a sequence chosen for its likelihood to be a good target. "An underlying aspect of the Joung paper is that the site that you are trying to target makes a difference," explains Lawson. "In our study, we biased ourselves toward sites that are favorable." modular assembly approach and facilitate the technology's adoption by small labs.

The technical choices in the two studies have led to ZFNs that differ in some waysspecificity, affinity and nuclease activityand this may explain a striking difference in the amount of RNA injected (Lawson injects 10-20 pg; Amacher uses 1-5 ng). Both groups have looked for off-target effects and found few or none. According to Amacher, even a small percentage of off-target mutations will not diminish the enthusiasm, as mutations would quickly segregate away from one another as long as they are not linked. "The zebrafish community would be ecstatic at the chance of getting a germline mutation in a gene of interest," she says.

Lawson concurs: "This will enable a lot of things that we could only have dreamed of doing 18 months ago." Looking forward, all see the same thing on the horizon: homologous recombination and the ability to start using the same technology in the zebrafish that others are using in the mouse.

\section{Veronique Kiermer}

\section{RESEARCH PAPERS}

Doyon Y. et al. Heritable targeted gene disruption in zebrafish using designed zinc-finger nucleases. Nat. Biotechnol. 26, 702-708 (2008).

Meng, X. et al. Targeted gene inactivation in zebrafish using engineered zinc-finger nucleases. Nat. Biotechnol. 26, 695-701 (2008).

Ramirez, C.L. et al. Unexpected failure rates for modular assembly of engineered zinc fingers. Nat. Methods 5, 374-375 (2008). 